\title{
Calibration of the top quark mass for Monte-Carlo event generators
}

\author{
Mathias Butenschoen, ${ }^{a}$ Bahman Dehnadi, ${ }^{b}$ André H. Hoang, ${ }^{c d}$ Vicent Mateu, ${ }^{e}$ \\ Moritz Preisser*c and lain W. Stewart ${ }^{f}$ \\ aII. Institut für Theoretische Physik, Universität Hamburg, \\ Luruper Chaussee 149, D-22761 Hamburg, Germany \\ ${ }^{b}$ Universität Siegen, Department Physik, Walter-Flex-Straße 3, D-57068 Siegen, Germany \\ ${ }^{c}$ University of Vienna, Faculty of Physics, Boltzmanngasse 5, A-1090 Wien, Austria \\ ${ }^{d}$ Erwin Schrödinger International Institute for Mathematical Physics, University of Vienna, \\ Boltzmanngasse 9, A-1090 Wien, Austria \\ e Departamento de Física Teórica and Instituto de Física Teórica, IFT-UAM/CSIC, \\ Universidad Autónoma de Madrid, Cantoblanco, 28049, Madrid, Spain \\ ${ }^{f}$ Center for Theoretical Physics, Massachusetts Institute of Technology, \\ Cambridge, MA 02139, USA \\ E-mail: mathias.butenschoen@desy · de,dehnadi@physik • uni-siegen.de, \\ andre.hoang@univie.ac.at, vicent.mateu@uam.es, \\ moritz.preisser@univie.ac.at, iains@mit.edu
}

\begin{abstract}
The most precise top quark mass measurements use kinematic reconstruction methods, determining the top mass parameter of a Monte Carlo event generator, $m_{t}^{\mathrm{MC}}$. Due to the complicated interplay of hadronization and parton shower dynamics in Monte Carlo event generators, relating $m_{t}^{\mathrm{MC}}$ to field theory masses is a non-trivial task. In this talk we present a calibration procedure to determine this relation using hadron level QCD predictions for 2-Jettiness in $e^{+} e^{-}$annihilation, an observable which has kinematic top mass sensitivity and has a close relation to the invariant mass of the particles coming from the top decay. The theoretical ingredients of the QCD prediction are explained. Fitting $e^{+} e^{-}$2-Jettiness calculations at NLL/NNLL order to PYTHIA 8.205, we find that $m_{t}^{\mathrm{MC}}$ agrees with the MSR mass at the scale $1 \mathrm{GeV}$ within uncertainties, $m_{t}^{\mathrm{MC}} \simeq m_{t, 1 \mathrm{GeV}}^{\mathrm{MSR}}$, but differs from the pole mass by $900 / 600 \mathrm{MeV}$.
\end{abstract}

38th International Conference on High Energy Physics 3-10 August 2016

Chicago, USA

${ }^{*}$ Speaker. 


\section{Introduction}

The most precise measurements of the top quark mass are based on direct reconstruction methods exploiting its kinematic properties and have reached uncertainties of about $0.5 \mathrm{GeV}[1,2,3]$. They are based on multivariate fits that use a maximum amount of information from the top decay final states. This includes template and matrix element fits for distributions such as the measured invariant mass. Since these observables are highly differential and depend on experimental cuts and details of the jet dynamics, multipurpose Monte Carlo (MC) event generators are employed in these analyses, and the measured mass is the top mass parameter $m_{t}^{\mathrm{MC}}$ contained in the particular $\mathrm{MC}$ event generator. Clearly, the interpretation of $m_{t}^{\mathrm{MC}}$ from the field theoretic point of view is influenced by the interplay of both perturbative and non-perturbative QCD effects and - because MC generators provide only approximate descriptions - may also depend in part on the MC tuning and the set of observables used in the analyses. In the direct reconstruction analyses referred to above the systematic uncertainties from MC modeling are a dominant part of the uncertainty budget, but they do not address in any way how $m_{t}^{\mathrm{MC}}$ is related to a mass parameter defined precisely in quantum field theory that can be globally used for higher order theoretical predictions. The relation is nontrivial because it requires an understanding of the interplay between the partonic components of the MC generator (hard matrix elements and parton shower) and the hadronization model. One can also say that - at the level of precision achieved for top mass measurements in direct reconstruction - MC generators should be considered as models whose partonic components and hadronization models are, through the tuning procedure, capable of describing experimental data to a precision that is higher than that of their partonic input.

In the past $m_{t}^{\mathrm{MC}}$ has frequently simply been identified with the pole mass, which can only be defined within perturbation theory. This is compatible with parton-shower implementations for massive quarks, but a direct identification is disfavored because of the sensitivity of $m_{t}^{\mathrm{MC}}$ to nonperturbative effects from below the MC shower cutoff $\Lambda_{c} \sim 1 \mathrm{GeV}$. Also, the pole mass has an $\mathscr{O}\left(\Lambda_{\mathrm{QCD}}\right)$ renormalon ambiguity, while $m_{t}^{\mathrm{MC}}$ does not, since information from perturbative QCD is not employed below $\Lambda_{c}$. It has been argued $[4,5]$ that $m_{t}^{\mathrm{MC}}$ has a closer relation to the MSR mass [6] $m_{t}^{\mathrm{MSR}}\left(R \approx \Lambda_{c}\right)$, where the scale $R$ defining this scheme is close to $\Lambda_{c}$.

For a given MC generator, $m_{t}^{\mathrm{MC}}$ can be calibrated with respect to a field theory mass scheme through a fit of $\mathrm{MC}$ predictions to hadron level QCD computations for observables closely related to the distributions that enter the experimental reconstruction analyses. In Ref. [7] we have provided a precise quantitative study on the interpretation of $m_{t}^{\mathrm{MC}}$ in terms of the MSR and pole mass schemes based on a hadron level prediction for the 2-Jettiness variable $\tau_{2}$ [8] for the production of a boosted top-antitop quark pair in $e^{+} e^{-}$annihilation. To be definite $\tau_{2}$ is defined as:

$$
\tau_{2}=1-\max _{\vec{n}_{t}} \frac{\sum_{i}\left|\vec{n}_{t} \cdot \vec{p}_{i}\right|}{Q}
$$

where the sum is over the 3-momenta of all final state particles, the maximum defines the thrust axis $\vec{n}_{\mathrm{t}}$ and $Q$ is the center of mass energy. In Refs. $[9,10]$ a factorization theorem has been proven for boosted top quarks, yielding hadron level predictions for $\tau_{2}$. The $\tau_{2}$ distribution has a distinguished peak very sensitive to the top mass, and is a delta function at $\tau_{2}^{\min }\left(m_{t}\right)=1-\sqrt{1-4 m_{t}^{2} / Q^{2}}$ at tree level. The peak region is dominated by dijet events where the top quarks decay inside narrow 
back-to-back cones, and $\tau_{2}$ is directly related to the sum of the squared invariant masses $M_{a, b}^{2}$ in the two hemispheres defined by the thrust axis $\vec{n}_{t},\left(\tau_{2}\right)_{\text {peak }} \approx\left(M_{a}^{2}+M_{b}^{2}\right) / Q^{2}[9,10]$. Therefore $\tau_{2}$ in the peak region is an observable with kinematic top mass sensitivity, just like those that enter the top quark mass reconstruction methods, and the results of the calibration study should provide information relevant for the interpretation of the direct reconstruction measurements.

\section{2-Jettiness Distribution}

The $\tau_{2}$ distribution in the peak region for boosted top quarks has the basic form

$$
\frac{\mathrm{d} \sigma}{\mathrm{d} \tau_{2}}=\int \mathrm{dk}\left(\frac{\mathrm{d} \hat{\sigma}_{\mathrm{s}}}{\mathrm{d} \tau_{2}}+\frac{\mathrm{d} \hat{\sigma}_{\mathrm{ns}}}{\mathrm{d} \tau_{2}}\right)\left(\tau_{2}-\frac{\mathrm{k}}{\mathrm{Q}}\right) \mathrm{F}_{\tau_{2}}(\mathrm{k})\left[1+\mathscr{O}\left(\frac{\Lambda_{\mathrm{QCD}}}{\mathrm{Q}}, \frac{\Gamma_{\mathrm{t}}}{\mathrm{m}_{\mathrm{t}}}\right)\right]
$$

where d $\hat{\sigma}_{\mathrm{s}} / \mathrm{d} \tau_{2}$ contains the singular partonic QCD corrections $\alpha_{s}^{j}\left[\ln ^{k}\left(\tau_{2}-\tau_{2}^{\min }\right) /\left(\tau_{2}-\tau_{2}^{\min }\right)\right]_{+}$ and $\alpha_{s}^{j} \delta\left(\tau_{2}-\tau_{2}^{\min }\right)$ in the dijet limit and $\mathrm{d} \hat{\sigma}_{\mathrm{ns}} / \mathrm{d} \tau_{2}$ stands for the remaining partonic nonsingular QCD corrections. The shape function $F_{\tau_{2}}$ describes non-perturbative effects from wide-angle soft gluon radiation [11]. The singular partonic contribution obeys the factorization theorem

$$
\begin{aligned}
& \frac{\mathrm{d} \hat{\sigma}_{\mathrm{s}}}{\mathrm{d} \tau_{2}}=Q H_{Q}^{(6)}\left(Q, \mu_{Q}\right) U_{H_{Q}}^{(6)}\left(Q, \mu_{Q}, \mu_{m}\right) H_{m}^{(6)}\left(Q, m_{t}, \mu_{m}\right) U_{H_{m}}^{(5)}\left(\frac{Q}{m_{t}}, \mu_{m}, \mu_{B}\right) \\
& \times \int \mathrm{ds} \int \mathrm{dk} J_{B, \tau_{2}}^{(5)}\left(\frac{s}{m_{t}}, \mu_{B}, \Gamma_{t}, \delta m_{t}\right) U_{S}^{(5)}\left(k, \mu_{B}, \mu_{S}\right) \hat{S}_{\tau_{2}}^{(5)}\left(Q\left[\tau_{2}-\tau_{2}^{\min }\left(m_{t}\right)\right]-\frac{s}{Q}-k, \mu_{S}\right),
\end{aligned}
$$

which is based on Soft-Collinear-Effective Theory $[12,13,14,15]$ and separates the contributions from the hard interactions in the hard functions $H_{Q}$ and $H_{m}$, the jet function $J_{B, \tau_{2}}$, and the soft cross-talk between the top and antitop jets in the partonic soft function $\hat{S}_{\tau}$. The jet function $J_{B, \tau_{2}}$ is derived in boosted HQET [9] since the collinear top jet invariant mass in the peak region is very close to the top quark mass. It includes the collinear dynamics of the decaying top quarks and leading top finite-width effects. The various evolution factors $U_{X}$ sum large logarithms.

Results for $\mathrm{d} \hat{\sigma}_{\mathrm{s}} / \mathrm{d} \tau_{2}$ with next-to-leading logarithmic resummation $+\mathscr{O}\left(\alpha_{s}\right)$ singular corrections (NLL + NLO) can be found in Ref. [10], with the addition of the virtual top quark contribution and rapidity logarithms in $H_{m}$ and $U_{H_{m}}$ from Ref. [16]. The $\mathrm{N}^{2} L L$ evolution in $U_{H_{Q}}$ and $U_{S}$ is known from the massless quark case, and is consistent with the direct $\mathscr{O}\left(\alpha_{s}^{2}\right)$ calculation of the $J_{B, \tau_{2}}$ anomalous dimension [17]. We implemented all the $\mathrm{N}^{2} \mathrm{LL}$ order ingredients for the proper treatment of the flavor number dependence [superscript (6) for including top as dynamic quark versus superscript (5) for excluding the top] in the RG evolution [18, 19]. We also include the $\mathscr{O}\left(\alpha_{s}\right)$ nonsingular corrections $\mathrm{d} \hat{\sigma}_{\mathrm{ns}} / \mathrm{d} \tau_{2}[20]$.

For the shape function $F_{\tau_{2}}$ we use the convergent basis functions introduced in Ref. [21] truncated to 4 elements (where the 4-th element is already numerically irrelevant), which determine the moments of the shape function $\Omega_{i}$ [22,23]. The leading power correction $\Omega_{1}$ is defined in the R-gap scheme such that it cancels an $\mathscr{O}\left(\Lambda_{\mathrm{QCD}}\right)$ renormalon present in $\hat{S}_{\tau_{2}}$ [24]. This renders $\Omega_{1}$ dependent on the subtraction scale $R_{S}$, and we quote results for $\Omega_{1}$ at the reference scales $R_{S}=2 \mathrm{GeV}$. Equation (2.2) is written in terms of a generic mass scheme $m_{t}$, with $\delta m_{t}=m_{t}^{\text {pole }}-m_{t}$ in $J_{B, \tau_{2}}^{(5)}$ controlling the dominant sensitivity to the mass scheme. In the pole mass scheme $\delta m_{t}=0$. Using renormalon-free schemes, the $\overline{\mathrm{MS}}$ mass with $\delta m_{t} \propto m_{t}$ is appropriate for the hard functions. In the 
jet function $J_{B, \tau_{2}}^{(5)}$ one has to adopt a scheme such as MSR [6] with $\delta m_{t} \sim R \sim \Gamma_{t}$ to avoid upsetting the power counting in the peak region. The evolution of the MSR mass with $R$ and of $\Omega_{1}$ with $R_{S}$ is described by R-evolution $[6,25]$. To sum large logarithms we use $\tau_{2}$-dependent scales $\mu_{i}\left(\tau_{2}\right)$ and $R_{i}\left(\tau_{2}\right)$, which can be expressed in terms of 9 parameters. These parameters are varied to estimate perturbative uncertainties.

For a given center of mass energy $Q$, the key parameters that enter the QCD factorization predictions for the $\tau_{2}$ distribution are the top mass $m_{t}$, the top width $\Gamma_{t}$, the hadronic parameters $\Omega_{i}$, and the strong coupling $\alpha_{s}\left(m_{Z}\right)$. We will consider fits both in the pole and the MSR mass schemes. The results in the MSR scheme are quoted in terms of $m_{t}^{\mathrm{MSR}}(1 \mathrm{GeV})$ following $[4,5]$.

\section{Fit Procedure}

For a given $m_{t}^{\mathrm{MC}}$ we produce MC datasets for $\mathrm{d} \sigma / \mathrm{d} \tau_{2}$ in the peak region for various $Q$ values. For a given profile and value of $\alpha_{s}\left(m_{Z}\right)$ we fit the parameters $m_{t}$ and $\Omega_{i}$ of the hadron level QCD predictions to this $\mathrm{MC}$ dataset. For each $Q$ value the distribution is normalized over the fit range, and multiple $Q$ s are needed simultaneously to break degeneracies. We construct the $\chi^{2}$-function using the statistical uncertainties in the MC datasets. We do the fit by first, for a given value of $m_{t}$, minimizing $\chi^{2}$ with respect to the $\Omega_{i}$ parameters. The resulting marginalized $\chi^{2}$ is then minimized with respect to $m_{t}$ used in the QCD predictions. Uncertainties obtained for the QCD parameters from this $\chi^{2}$ simply reflect the MC statistical uncertainties used to construct the $\chi^{2}$. To estimate the perturbative uncertainty in the QCD predictions we take 500 random points in the profilefunction parameter space and perform a fit for each of them. The 500 sets of best-fit values provide an ensemble from which we remove the upper and lower $1.5 \%$ in the mass values to eliminate potential numerical outliers. From this we then determine central values by averaging the largest and smallest values and perturbative uncertainties from half the covered interval.

To illustrate the calibration procedure we use PYTHIA $8.205[26,27]$ with the $e^{+} e^{-}$default tune 7 (the Monash 2013 tune [28] for which $\Lambda_{c}=0.5 \mathrm{GeV}$ ) for top mass parameter values $m_{t}^{\mathrm{MC}}=170,171,172,173,174$ and $175 \mathrm{GeV}$. We use a fixed top quark width $\Gamma_{t}=1.4 \mathrm{GeV}$ which is independent of $m_{t}^{\mathrm{MC}}$. No other changes are made to the default settings. To minimize statistical uncertainties we generate each distribution with $10^{7}$ events. We have carried out fits for the following seven Q sets (in GeV units): (600,1000,1400), (700,1000,1400), (800,1000, 1400), (600 - 900), (600-1400), (700 - 1000) and (700 - 1400), where the ranges refer to steps of 100. For each one of these sets we have considered three ranges of $\tau_{2}$ in the peak region: $(60 \%, 80 \%)$, $(70 \%, 80 \%)$ and $(80 \%, 80 \%)$, where $(x \%, y \%)$ means that we include regions of the spectra whose $\tau_{2}<\tau_{2}^{\text {peak }}$ having cross-section values larger than $x \%$ of the peak height, and $\tau_{2}>\tau_{2}^{\text {peak }}$ with cross sections larger than $y \%$ of the peak height, where $\tau_{2}^{\text {peak }}$ is the peak position. This makes a total of 21 fit settings each of which gives central values and scale uncertainties for the top mass and the $\Omega_{i}$.

\section{Numerical Results of the Calibration}

To visualize the stability of our fits we display in Fig. 1 the distribution of best-fit mass values obtained for 500 random profile functions for $m_{t}^{\mathrm{MC}}=173 \mathrm{GeV}$ based on the $Q$ set $(600-1400)$ 

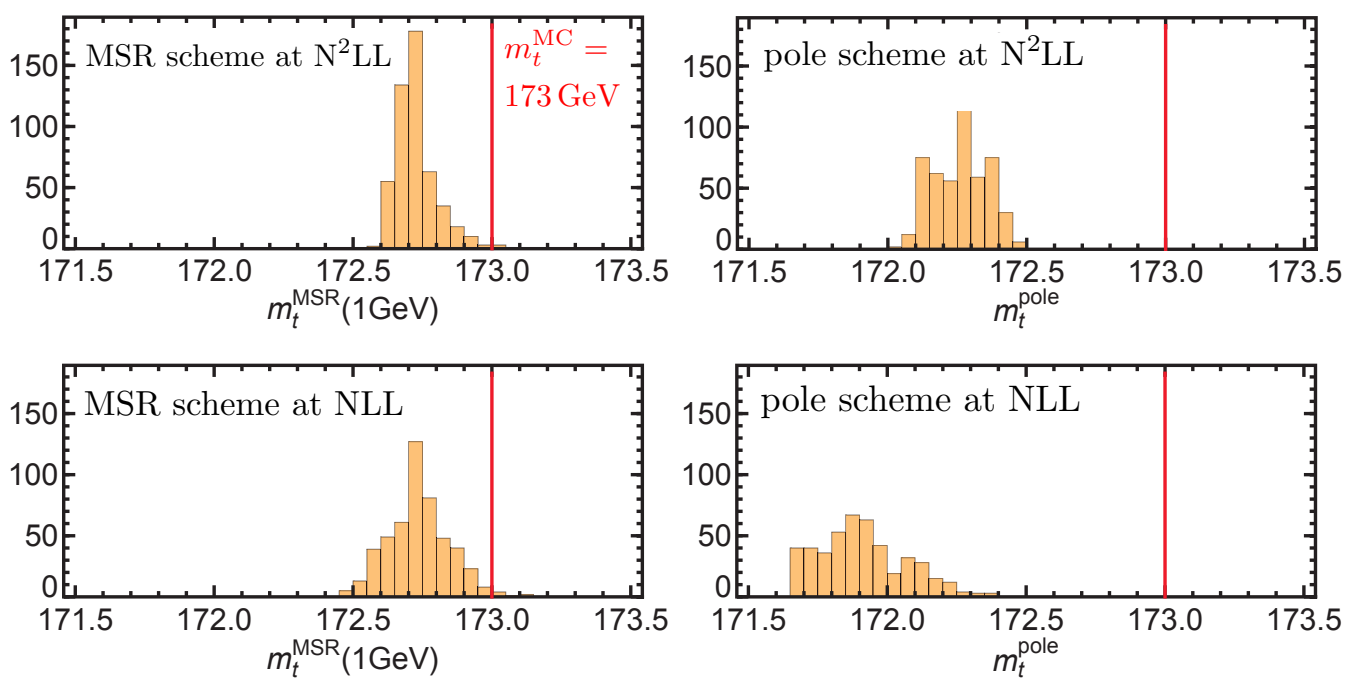

Figure 1: Distribution of best-fit mass values from the scan over parameters describing perturbative uncertainties. Results are shown for cross sections employing the MSR mass $m_{t}^{\mathrm{MSR}}(1 \mathrm{GeV})$ (left) and the pole mass $m_{t}^{\text {pole }}$ (right), both at $\mathrm{N}^{2} \mathrm{LL}$ and NLL. The PYTHIA datasets use $m_{t}^{\mathrm{MC}}=173 \mathrm{GeV}$ as an input.

and the bin range $(60 \%, 80 \%)$. Results are shown for $m_{t}^{\mathrm{MSR}}(1 \mathrm{GeV})$ and $m_{t}^{\text {pole }}$ at NLL and $\mathrm{N}^{2} \mathrm{LL}$ order, exhibiting good convergence, with the higher order result having a smaller perturbative scale uncertainty. The results for $m_{t}^{\mathrm{MSR}}(1 \mathrm{GeV})$ are stable and about $200 \mathrm{MeV}$ below $m_{t}^{\mathrm{MC}}$ confirming the close relation of $m_{t}^{\mathrm{MSR}}(1 \mathrm{GeV})$ and $m_{t}^{\mathrm{MC}}$ suggested in Refs. [4, 5]. We observe that $m_{t}^{\mathrm{pole}}$ is about $1.1 \mathrm{GeV}(\mathrm{NLL})$ and $0.7 \mathrm{GeV}\left(\mathrm{N}^{2} \mathrm{LL}\right)$ lower than $m_{t}^{\mathrm{MC}}$, demonstrating that corrections here are bigger, and that the MC mass cannot simply be identified with the pole mass. The results from the fits to the 21 different $\mathrm{Q}$ sets and bin ranges mentioned above are quite similar. Their differences can be interpreted as a quantification of the level of incompatibility between the MC event generator results and the QCD predictions. Unlike the perturbative uncertainties they need not necessarily to decrease when going from NLL to $\mathrm{N}^{2} \mathrm{LL}$. We therefore use the differences from the 21 fits to assign an additional incompatibility uncertainty between QCD and the MC generator for the calibration.

To quote final results we used the following procedure: (1) Take the average of the highest and lowest central values from the 21 sets as the final central value of our calibration. (2) Take the average of the scale uncertainties of these sets as our final estimate for the perturbative uncertainty. (3) Take the half of the difference of the largest and smallest central values from the sets as the incompatibility uncertainty between QCD and the MC. (4) Quadratically add the perturbative, and incompatibility errors to obtain a final uncertainty.

Using $\alpha_{s}$ values within the uncertainty of the world average $\alpha_{s}\left(m_{Z}\right)=0.1181(13)$ gives an additional parametric uncertainty of $\simeq 20 \mathrm{MeV}$ for $m_{t}^{\mathrm{MSR}}(1 \mathrm{GeV})$ and $m_{t}^{\text {pole }}$ at $\mathrm{N}^{2} L L$ order. This is an order of magnitude smaller than the other uncertainties and we therefore neglect it. Table 1 shows our final results for the MSR mass $m_{t}^{\mathrm{MSR}}(1 \mathrm{GeV})$ and $m_{t}^{\text {pole }}$ at NLL and $\mathrm{N}^{2} \mathrm{LL}$ order, utilizing the $m_{t}^{\mathrm{MC}}=173 \mathrm{GeV}$ dataset. For $m_{t}^{\mathrm{MSR}}(1 \mathrm{GeV})$ we observe a reduction of perturbative uncertainties from $260 \mathrm{MeV}$ at NLL to $190 \mathrm{MeV}$ at $\mathrm{N}^{2} \mathrm{LL}$. The corresponding incompatibility un- 


\begin{tabular}{|lllccc|}
\multicolumn{7}{c|}{$m_{t}^{\mathrm{MC}}=173 \mathrm{GeV}\left(\tau_{2}^{e^{+}} e^{-}\right.$} \\
\hline mass & order & central & perturb. & incompatibility & total \\
\hline \hline$m_{t, 1 \mathrm{GeV}}^{\mathrm{MSR}}$ & $\mathrm{NLL}$ & 172.80 & 0.26 & 0.14 & 0.29 \\
$m_{t, 1 \mathrm{GR}}^{\mathrm{MSR}}$ & $\mathrm{N}^{2} \mathrm{LL}$ & 172.82 & 0.19 & 0.11 & 0.22 \\
$m_{t}^{\text {pole }}$ & $\mathrm{NLL}$ & 172.10 & 0.34 & 0.16 & 0.38 \\
$m_{t}^{\text {pole }}$ & $\mathrm{N}^{2} \mathrm{LL}$ & 172.43 & 0.18 & 0.22 & 0.28 \\
\hline
\end{tabular}

Table 1: Results of the calibration for $m_{t}^{\mathrm{MC}}=173 \mathrm{GeV}$ in PYTHIA, combining results from all Q sets and bin ranges. Shown are central values, perturbative and incompatibility uncertainties, and the total uncertainty, all in $\mathrm{GeV}$

certainties are 140 and $110 \mathrm{MeV}$. The corresponding fit results for the first shape function moment are $\Omega_{1}^{\mathrm{PY}}=0.42 \pm 0.07 \pm 0.03 \mathrm{GeV}$ at $\mathrm{N}^{2} \mathrm{LL}$ and $\Omega_{1}^{\mathrm{PY}}=0.41 \pm 0.07 \pm 0.02 \mathrm{GeV}$ at NLL order with the first uncertainty coming from scale variation and second from incompatibility. The result agrees nicely with the expectation that $\Omega_{1} \sim \Lambda_{\mathrm{QCD}}$. For $m_{t}^{\text {pole }}$ there is a significant difference to $m_{t}^{\mathrm{MC}}$, and we observe that the central value shifts by $330 \mathrm{MeV}$ between NLL and $\mathrm{N}^{2} \mathrm{LL}$ order. There is a reduction of perturbative uncertainties like in the MSR scheme, however the incompatibility uncertainty increases at $\mathrm{N}^{2} \mathrm{LL}$ order. These results may not be unexpected, since the pole mass often leads to poorer convergence of perturbative series.

We have carried out the calibration procedure for $m_{t}^{\mathrm{MC}}$ values between 170 and $175 \mathrm{GeV}$ in steps of $1 \mathrm{GeV}$, and the outcome of our fits showed a behavior consistent with the results given in Tab. 1. In future studies such calibration results should be independently determined for different $\mathrm{MC}$ event generators and also for generator settings (such as different tunes).

To the extent that the treatment of the top in MC generators and QCD factorizes for different kinematically sensitive observables and from whether one considers $e^{+} e^{-}$or $p p$ collisions, our method can be used to calibrate $m_{t}^{\mathrm{MC}}$ in current experimental reconstruction analyses. $p p$ collisions introduce initial state radiation, color reconnection, and additional hadronization and multi-parton interaction effects, not present in $e^{+} e^{-}$. In the future our method can be extended to use a $p p$ observable to directly study these effects. Prior to this, we believe that applying our $e^{+} e^{-}$calibration to $m_{t}^{\mathrm{MC}}$ from a typical $p p$ reconstruction analysis will give a more accurate result than simply assuming $m_{t}^{\mathrm{MC}}=m_{t}^{\text {pole }}$. The calibration may also provide new ways to test and improve MC event generators.

\section{References}

[1] Tevatron Electroweak Working Group collaboration, CDF and D0, Combination of CDF and DO results on the mass of the top quark using up to $9.7 \mathrm{fb}^{-1}$ at the Tevatron, 1407.2682.

[2] CMS collaboration, V. Khachatryan et al., Measurement of the top quark mass using proton-proton data at $\sqrt{(s)}=7$ and 8 TeV, Phys. Rev. D93 (2016) 072004, [1509.04044].

[3] ATLAS collaboration, M. Aaboud et al., Measurement of the top quark mass in the $t \bar{t} \rightarrow$ dilepton channel from $\sqrt{s}=8 \mathrm{TeV}$ ATLAS data, Phys. Lett. B761 (2016) 350-371, [1606.02179].

[4] A. H. Hoang and I. W. Stewart, Top Mass Measurements from Jets and the Tevatron Top-Quark Mass, Nucl. Phys. Proc. Suppl. 185 (2008) 220-226, [0808.0222]. 
[5] A. H. Hoang, The Top Mass: Interpretation and Theoretical Uncertainties, in 7th International Workshop on Top Quark Physics (TOP2014) Cannes, France, September 28-October 3, 2014, 2014. 1412.3649.

[6] A. H. Hoang, A. Jain, I. Scimemi and I. W. Stewart, Infrared Renormalization Group Flow for Heavy Quark Masses, Phys. Rev. Lett. 101 (2008) 151602, [0803.4214].

[7] M. Butenschoen, B. Dehnadi, A. H. Hoang, V. Mateu, M. Preisser and I. W. Stewart, Top Quark Mass Calibration for Monte Carlo Event Generators, 1608.01318.

[8] I. W. Stewart, F. J. Tackmann and W. J. Waalewijn, N-Jettiness: An Inclusive Event Shape to Veto Jets, Phys.Rev.Lett. 105 (2010) 092002, [1 004 . 248 9].

[9] S. Fleming, A. H. Hoang, S. Mantry and I. W. Stewart, Jets from massive unstable particles: Top-mass determination, Phys. Rev. D77 (2008) 074010, [hep-ph/ 0703207 ].

[10] S. Fleming, A. H. Hoang, S. Mantry and I. W. Stewart, Top Jets in the Peak Region: Factorization Analysis with NLL Resummation, Phys. Rev. D77 (2008) 114003, [0 711.2079$].$

[11] G. P. Korchemsky and G. Sterman, Power corrections to event shapes and factorization, Nucl. Phys. B555 (1999) 335-351, [hep-ph/9902341].

[12] C. W. Bauer, S. Fleming and M. E. Luke, Summing Sudakov logarithms in $B \rightarrow X_{s} \gamma$ in effective field theory, Phys. Rev. D63 (2000) 014006, [hep-ph/ 0005275$].$

[13] C. W. Bauer, S. Fleming, D. Pirjol and I. W. Stewart, An effective field theory for collinear and soft gluons: Heavy to light decays, Phys. Rev. D 63 (2001) 114020, [hep-ph / 0011336 ].

[14] C. W. Bauer and I. W. Stewart, Invariant operators in collinear effective theory, Phys. Lett. B 516 (2001) 134-142, [hep-ph/0107001].

[15] C. W. Bauer, D. Pirjol and I. W. Stewart, Soft-Collinear Factorization in Effective Field Theory, Phys. Rev. D65 (2002) 054022, [hep-ph/ 0109045$].$

[16] A. H. Hoang, A. Pathak, P. Pietrulewicz and I. W. Stewart, Hard Matching for Boosted Tops at Two Loops, JHEP 12 (2015) 059, [1508.04137].

[17] A. Jain, I. Scimemi and I. W. Stewart, Two-loop Jet-Function and Jet-Mass for Top Quarks, Phys. Rev. D77 (2008) 094008, [0 801.0743$].$

[18] S. Gritschacher, A. H. Hoang, I. Jemos and P. Pietrulewicz, Secondary Heavy Quark Production in Jets through Mass Modes, Phys. Rev. D88 (2013) 034021, [1302 . 4743].

[19] P. Pietrulewicz, S. Gritschacher, A. H. Hoang, I. Jemos and V. Mateu, Variable Flavor Number Scheme for Final State Jets in Thrust, Phys. Rev. D90 (2014) 114001, [1405.4860].

[20] B. Dehnadi. PhD thesis, University of Vienna, June, 2016.

[21] Z. Ligeti, I. W. Stewart and F. J. Tackmann, Treating the b quark distribution function with reliable uncertainties, Phys. Rev. D78 (2008) 114014, [0 807 .1926].

[22] R. Abbate, M. Fickinger, A. H. Hoang, V. Mateu and I. W. Stewart, Thrust at $N^{3} L L$ with Power Corrections and a Precision Global Fit for $\alpha_{s}\left(m_{Z}\right)$, Phys. Rev. D83 (2011) 074021, [1 006.3080 ].

[23] R. Abbate, M. Fickinger, A. H. Hoang, V. Mateu and I. W. Stewart, Precision Thrust Cumulant Moments at $N^{3}$ LL, Phys. Rev. D86 (2012) 094002, [1204. 5746].

[24] A. H. Hoang and I. W. Stewart, Designing Gapped Soft Functions for Jet Production, Phys. Lett. B660 (2008) 483-493, [0 709 . 3519]. 
[25] A. H. Hoang, A. Jain, I. Scimemi and I. W. Stewart, R-evolution: Improving perturbative QCD, Phys. Rev. D82 (2010) 011501, [0908.3189].

[26] T. Sjöstrand, S. Mrenna and P. Z. Skands, PYTHIA 6.4 Physics and Manual, JHEP 05 (2006) 026, [hep-ph/0603175].

[27] T. Sjöstrand, S. Ask, J. R. Christiansen, R. Corke, N. Desai, P. Ilten et al., An Introduction to PYTHIA 8.2, Comput. Phys. Commun. 191 (2015) 159-177, [1410.3012].

[28] P. Skands, S. Carrazza and J. Rojo, Tuning PYTHIA 8.1: the Monash 2013 Tune, Eur. Phys. J. C74 (2014) 3024, [1404.5630]. 